\title{
Targeted drug delivery to the brain using magnetic nanoparticles
}

\begin{abstract}
Brain capillary endothelial cells denote the blood-brain barrier (BBB), and conjugation of nanoparticles with antibodies that target molecules expressed by these endothelial cells may facilitate their uptake and transport into the brain. Magnetic nanoparticles can be encapsulated in liposomes and carry large molecules with therapeutic potential, for example, siRNA, cDNA and polypeptides. An additional approach to enhance the transport of magnetic nanoparticles across the BBB is the application of extracranially applied magnetic force. Stepwise targeting of magnetic nanoparticles to brain capillary endothelial cells followed by transport through the BBB using magnetic force may prove a novel mechanism for targeted therapy of macromolecules to the brain.
\end{abstract}

For decades, the blood-brain barrier (BBB) has both fascinated and challenged the biomedical research community. Fascinated, because it exhibits a dramatic impermeability to solutes of the blood plasma in spite of consisting only a single layer of endothelial cells with adjacent tight junctions, hence revealing a transendothelial electric resistance of more than $2000 \mathrm{Ohm} / \mathrm{cm}^{2}[1,2]$; and challenged as it only allows the entry of drugs that possess the characteristics of being smaller than 400-600 Da, unless being highly hydrophobic [1-3]. Further complicating the possibilities for passaging drugs to the brain, the brain endothelial cells denoting the $\mathrm{BBB}$ profoundly express multidrug resistance proteins that preclude the entrance of several lipophilic molecules in a size independent manner $[1,2,4,5]$. In consequence, current drugs available for therapy to the brain denote highly lipophilic molecules, for example, hypnotics and anesthetics, which easily pass the BBB via diffusion, or small and hydrophilic molecules that enter the brain via facilitated transport due to their structural similarity with endogenously expressed nutrient transporters, for example, selective serotonin and noradrenalin reuptake inhibitors (cf. [5]). The restraints in drug access to the brain hence account for several chal- lenges concerning drug delivery, and further exploration of the BBB impermeability issue is pertinent.

The issue of improved drug delivery to the brain is also relevant, as novel methods could enable transport of therapeutic molecules, large and hydrophilic in nature or otherwise incapable of passing the BBB $[4,5]$. The group of large hydrophilic molecules fits the concept of 'biopharmaceuticals' and encompasses therapeutic polypeptides, recombinant proteins, monoclonal antibodies and genetic molecules based on antisense technology such as cDNA, siRNA and miRNA [6].

One strategy to enable transport of biopharmaceuticals into the brain is to utilize nanoparticles encapsulated with large and hydrophilic molecules. Specific antibodies targeting the transferrin receptor attached to the surface of the nanoparticles may further allow their targeted delivery at the BBB $[4,5]$. However, this targeting approach will limit the nanoparticles to enter the brain endothelial cells without further passage into the brain, unless the specific antibodies are designed to limit their binding affinity for the transferrin receptor [7]. nanoparticles to pass from the brain endothelial cells and further into the brain are
Therefore, novel approaches to enable
Louiza Bohn Thomsen*,1, Maj Schneider Thomsen ${ }^{1}$ \& Torben Moos ${ }^{1}$

'Laboratory of Neurobiology, Section of Biomedicine, Department of Health Science \& Technology, Aalborg University, Fredrik Bajers Vej 3 B, 9220 Aalborg East, Denmark *Author for correspondence: lbt@hst.aau.dk 


\section{Key terms}

Blood-brain barrier: The barrier between the bloodstream and the brain parenchyma, which is impermeable to most drugs and therefore an obstacle for drug delivery to the brain.

Magnetic nanoparticles: Nanoparticles consisting of a magnetic compound, often iron oxide, with an ability to be magnetized by a magnetic field.

Magnetic targeting: Magnetic nanoparticles can be moved toward a target by a magnetic field.

Magnetoliposomes: Magnetic nanoparticles encapsulated in the aqueous inner compartment of liposomes resulting in liposomes, which can be magnetically guided.

Super paramagnetic oxide nanoparticles: Small nanoparticles consisting of iron oxide with high magnetic susceptibility. In the absence of an external magnet, the SPIONs have a magnetization of zero. Only when a magnetic field is applied, the SPIONs are magnetized.

highly warranted. Application of an external magnetic field is a novel approach that could allow guidance of magnetic nanoparticles across the BBB [8-11].

The magnetic nanoparticles are composed of an iron oxide core most often magnetite $\left(\mathrm{Fe}_{3} \mathrm{O}_{4}\right)$ or maghemite $\left(\gamma-\mathrm{Fe}_{2} \mathrm{O}_{3}\right)$. This iron oxide core is a main feature, enabling movement in a magnetic field. Magnetic nanoparticles are currently being used for a variety of in vivo and in vitro purposes, for example, as contrast agents for MRI [12], treatment in hyperthermia [13], cell labeling and separation [14-16], magnetofection [17] and drug delivery by magnetic targeting [18-23]. For such purposes the magnetic nanoparticles can be polymer coated, but they can also be encapsulated inside liposomes, hence forming magnetoliposomes $[12,24,25]$

In this review, we cover the perspectives of using magnetic nanoparticles for guided uptake and transport across the BBB by using application of external magnetic force. We place emphasis on the composition and permeability restraints of the BBB and its immediate surroundings called the neurovascular unit (NVU), which is composed of pericytes enwrapped in a basement membrane and astrocytes that invest this basement membrane from its outer surface (Figure 1). We describe the principles of using molecular targeting at the BBB as an alternative or supplement to magnetic guidance, and we discuss the potential of combining targeted delivery of magnetic nanoparticles to the brain with the application of an external magnetic field.

\section{The BBB \& the neurovascular unit}

Neurons are fragile cells with a high dependency on the homeostatic mechanisms that regulate their microenvironment. Nonfenestrated brain endothelial cells and their intermingling tight junctions define the $\mathrm{BBB}$ that contributes significantly to the extracellular environment of the brain via regulation of the influx of ions and nutrients, while simultaneously protect- ing against the entry of many toxic substances [2]. The brain endothelial cells interconnect through tight junction and adherens junction proteins. The tight junctions restrict virtually any paracellular passage of ions, polar solutes and macromolecules through the BBB $[1,5,26]$. The adherens junctions structurally interconnect the brain endothelial cells and have a major role in formation of tight junctions influenced by pericytes and astrocytes [27-30]. The tight junctions restrict almost all paracellular passage of ions, polar solutes and macromolecules [1,5,26]. Magnetic nanoparticles and liposomes in diameters of $70 \mathrm{~nm}$ do not pass the $\mathrm{BBB}[31,32]$.

Pericytes and astrocytes surround the endothelial cells and these cells are only separated from each other by a basement membrane (Figure 1). The basement membrane completely enwraps the pericytes, while astrocytic end-feet contact the basement membrane from the outer surface (Figure 1). The basement membrane contains proteins such as laminins, collagen IV, nidogens and heparin sulfate proteoglycans [33,34]. The pericytes and astrocytes influence the brain endothelial cells to induce and maintain their barrier properties via stimulation of tight junction protein synthesis [2730]. Astrocytic end-feet invest approximately $99 \%$ of the outer basement membrane and likely affect the functioning of the brain endothelial cells, including their barrier properties, protein internalization and vesicle trafficking via secreted molecules that diffuses upstream to target receptors expressed by the brain endothelial cells on their abluminal side [35].

The basement membrane protein mesh form a tight layer that has been shown to be capable of halting magnetic nanoparticles after osmotic opening of the BBB leading to perivascular diffusion of molecules of the blood plasma into the brain [32]. The basement membrane is not a restraint for further diffusion into the brain of large proteins such as targeted bispecific antibodies passing through the intact brain endothelial cells via transcellular transport [36-38], or albumin passing a perturbed BBB by paracellular transport, for example, in conditions with osmotic opening [39].

\section{Molecular targeting at the BBB}

The extreme limitations in the BBB permeability to large or hydrophilic molecules with therapeutic potentials call for novel approaches to enable transport to the brain. The principle of making molecules lipophilic to enable their diffusion across the cell membrane is limited to nonpolar, lipid-soluble and/or small molecules, and many if not all of the next generation of therapeutics included in the concept of biopharmaceuticals (see 'Introduction' section) fail to include. Increasing the lipophilicity does not increase the quantitative uptake 


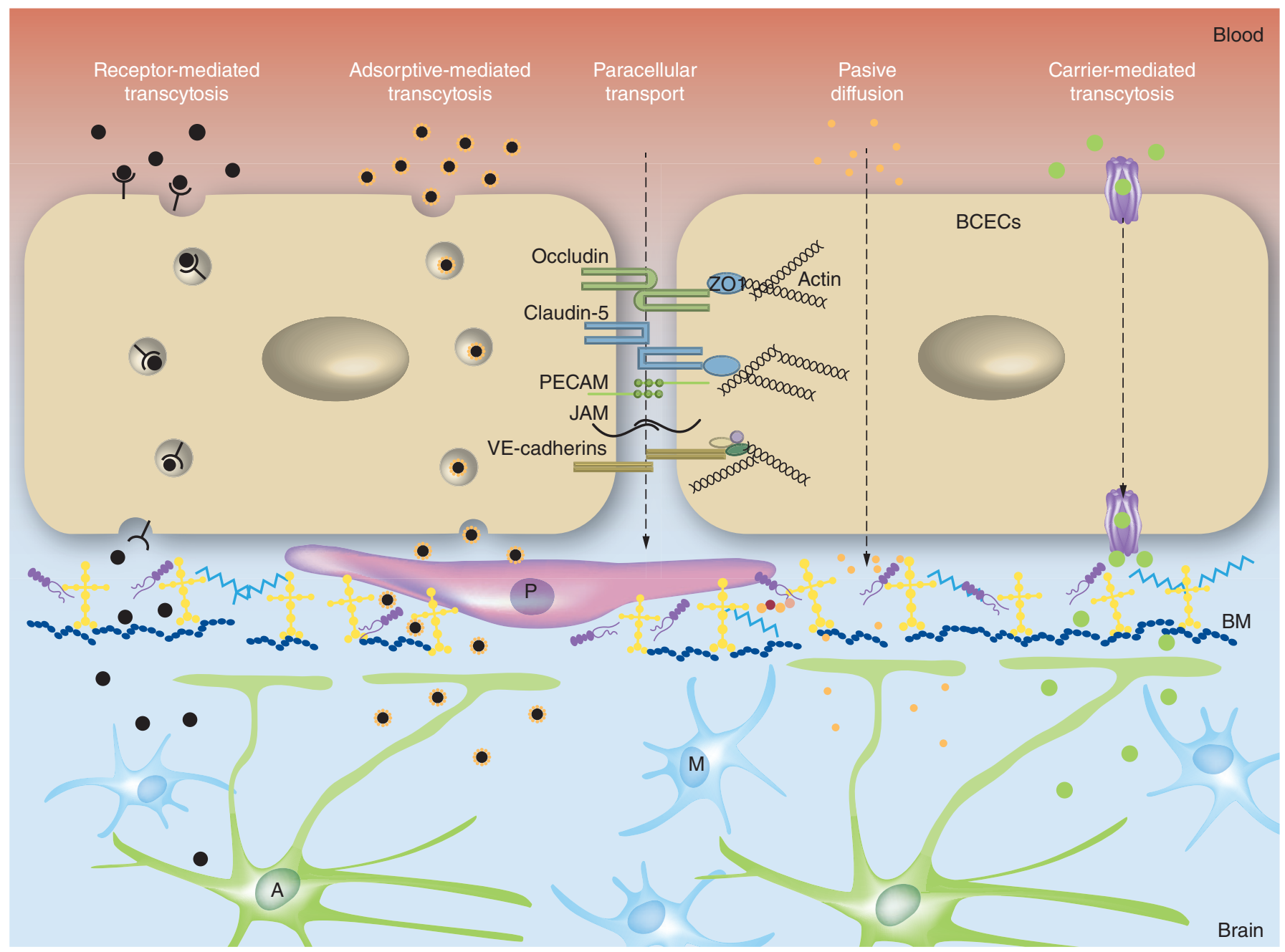

Figure 1. Illustration depicting different transport routes through the blood-brain barrier and the neurovascular unit. Molecules may access the brain from the bloodstream via paracellular transport or four different transendothelial transportation routes: receptor mediated transcytosis, adsorptive mediated transcytosis, passive diffusion and carrier-mediated transcytosis [26].

A: Astrocyte; BCEC: Brain capillary endothelial cells; BM: Basement membrane; M: Microglia; P: Pericyte.

Reproduced with permission from @ C Current Medicinal Chemistry (2014).

in the brain unless provided in much larger doses, as the high lipophilicity leads to a rapid clearance from the circulation and accumulation nonspecifically everywhere in the body (cf. [5]). Furthermore, the prominent expression of a number of ATP-binding cassette (ABC) efflux transporters are present on the abluminal and luminal membranes of the brain endothelial cells [40]. In extreme contrast to the small lipophilic molecules, the large molecules are unlikely to pass cellular membranes unless the recipient cell provides a machinery containing receptor-mediated endocytosis and subsequent transcytosis. The brain endothelial cells provide such machinery to enable receptor-mediated uptake of naturally occurring ligands carrying important nutrients such as glucose (insulin receptor) and iron (transferrin receptor). Whereas there is a common agreement that such receptors are expressed by brain endothelial cells, it is far from acknowledged that the receptormediated uptake of insulin or transferrin is followed by transcytotic transport into the brain (cf. [5]).

Interestingly, among the endothelial cells lining the vessels in the body only those of the brain express transferrin receptors. This makes the transferrin receptor particularly relevant for targeting purposes [37,41-44]. For drug delivery purposes, employment of antibodies against the transferrin receptor is preferable, as they do not compete with naturally occurring transferrin secreted from the liver. The antibodies directed against the transferrin receptor bind to this receptor leading to blood-to-endothelium transport [31,37]. The antitransferrin receptor antibodies are then internalized and transported to the endosomal-lysosomal system [45]. It is not evident whether the transferrin receptor-containing endosomes presents themselves on the abluminal 
membrane of the brain endothelial cells, which would be indicative of transcytosis [45]. Antibodies against the transferrin receptor may however transverse the brain endothelial cells if the binding affinity for the receptor is reduced by design [31]. This will allow for the essential 'endothelium-to-brain transport' and release of the antibodies inside the brain interstitium hence allowing the antibodies to following the entire route from blood to brain [31]. Transferrin receptors are also expressed by neurons $[46,47]$. The affinity-modified antibodies may thus undergo receptor-mediated endocytosis in neurons subsequent to their transport across the BBB. Conjugation of antitransferrin receptor antibodies to the surface of nanoparticles leads to uptake inside brain endothelial cells, but the particles remain within the endothelial cells without further transport into the brain [31].

The brain endothelial cells also have a high activity of catabolic enzymes, which means that the brain endothelial cells either directly pump lipophilic substances out of the brain endothelial cells, or they catabolize and subsequently efflux metabolites of lipophilic substances that succeed in passing into the brain endothelial cells [48].

\section{The use of magnetic nanoparticles for biological purposes}

Magnetic nanoparticles can be divided into two subdivisions, in other words, paramagnetic nanoparticles (PMNPs) and super paramagnetic iron oxide nanoparticles (SPIONs). The PMNPs are larger than $100 \mathrm{~nm}$ but still within the nanometer scale and the SPIONs are less than $100 \mathrm{~nm}$. SPIONs with a size of less than $50 \mathrm{~nm}$ are also referred to as ultrasuper paramagnetic iron oxide nanoparticles (USPIONs) [19,49,50]. The physical properties of SPIONs are of high importance, because they allow the particles to become magnetic with a high magnetic susceptibility in the presence of an external magnet as opposed to the much weaker magnetic susceptibility of PMNPs that have a larger diameter [19]. This feature makes SPIONs highly attractive for drug delivery purposes, because they are more easily attracted toward a magnet over large distances in comparison with the PMNPs [19]. SPIONs are good candidate for both targeting purposes and conjugation with biotherapeutics, as they can retain within tissues for long and still undergo biocompatible degradation [51,52]. They can also be encapsulated within liposomes (Figure 2) [51,52].

When administered into the circulation, magnetic nanoparticles are at risk of being taken up by phagocytic cells and cleared by the reticulo-endothelial system (RES). Surface modifications are therefore made to increase their biocompatibility and systemic half- life [53-55]. The iron oxide core of magnetic nanoparticles can be coated with a variety of different biocompatible matrices. A surface coat often consists of polymers, for example, starch or chitosan, which affect the particle charge and size, and thereby also the systemic half-life and the rate of uptake of the nanoparticles in brain endothelial cells [19]. Negatively charged, starch-coated SPIONs with a diameter of $117 \mathrm{~nm}$ are taken up by brain endothelial cells in vitro (Figure 3) [8]. The surface coat can also be utilized for functionalization (Figure 2). Polyethylene glycol (PEG) can be inserted into the surface coat. It reduces protein absorption and increases the circulation time of nanoparticles due to a response from the innate immune system [56,57]. The surface coat also enables attachment of ligands or monoclonal antibodies such as antitransferrin receptor antibodies with affinity for brain endothelial cells. This feature may direct the SPIONSs toward specific uptake of the SPIONs in brain endothelial cells when injected into the circulation, as it may decrease their side effects by decreasing delivery to nontarget organs [58].

The toxicity of SPIONs is low as revealed from several studies $[8,25,59,60]$, which is partly due to their iron oxide core that gets degraded to iron containing lowmolecular-weight substances that can be cleared by brain macrophages and microglia. Theoretically, these iron-containing substances are also cleared inside the brain by the neurons, and this could be toxic to the neurons as iron occurring on ferrous form is highly oxidative active and could ultimately cause neurodegeneration. This notion also gain support from that astrocytes in culture are intoxicated by SPIONs [61]. On the other hand, the brain gains more iron as part of the normal gain. Likewise, we were unable to detect toxic effects on brain endothelial cells exposed to SPIONs in vitro [8], but clearly the possible toxicity of the iron-containing SPIONs needs more attention. The iron of the degraded SPIONs would be presumed to undergo incorporation in the body's iron stores $[19,62]$. Whether the SPIONs will exert toxicity also seems to be very dependent upon which cell type is examined in a combination of the polymer coat, charge and concentration of SPIONs $[19,59,63]$. The cytotoxicity of three types of SPIONs; naked SPIONs, SPIONs-COOH and SPIONs- $\mathrm{NH}_{2}$ were tested on three types of cell lines; human cardiac myocytes (HCM), human embryonic kidney cells (293T) and human neuroblastoma cells (BE-2-C) [63]. The human neuroblastoma cells were most prone to toxic damage induced by SPIONs compared with the heart and kidney cells [63]. Primary neurons and astrocytes were more sensitive to positively aminosilane-coated SPIONs than negatively charged AMS-SPIONs [60], which indicate that the cell response toward SPIONs can be very dif- 


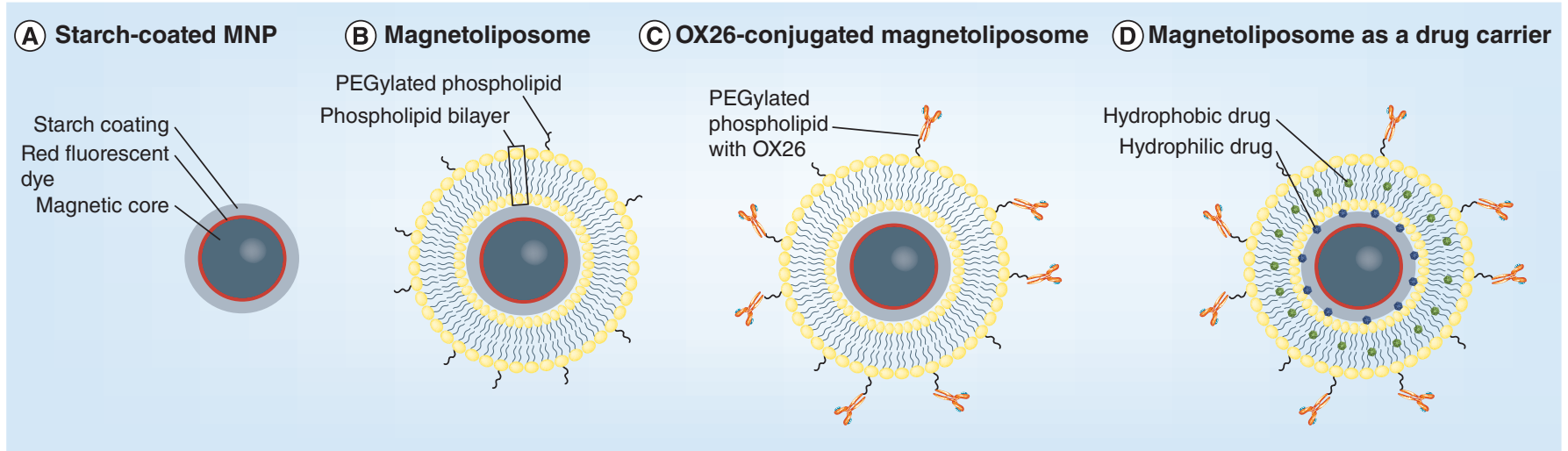

Figure 2. Illustration showing the composition of a magnetic nanoparticle (A), a magneto-liposome (B), a magneto-liposome with a targeting molecule (C) and a magneto-liposome with a targeting molecule loaded with cargo (D). The magnetic nanoparticle (A) consists of a magnetic core covered by a fluorescent coat, which is covered by a polymer coat. The magnetoliposome (B) consists of a double-layered liposome which is formed around a magnetic nanoparticle and has been functionalized with polyethylene glycol (PEG). Functionalized magneto-liposome (C) with a targeting molecule (the monoclonal antibody, OX26) attached to PEG on the surface of the magneto-liposome. Functionalized magneto-liposome (D) showing how hydrophobic cargo can be loaded in the phospholipid bilayer (green) and hydrophilic substances encapsulated in the fluid-filled inner compartment (blue) together with a magnetic nanoparticle.

ferent depending on cell type and the cell specific coping mechanisms. Negatively charged SPIONs induce a lower cytotoxic response than positively charged SPIONs and the naked SPIONs [63]. Both negatively and positively charged AmS-SPIONs were nontoxic below a concentration of $100 \mu \mathrm{g} / \mathrm{ml}$ when administered to
bEnd.3, primary astrocytes or primary neurons [60]. Starch-coated SPIONs added to human brain micro endothelial cells in a concentration of $140 \mu \mathrm{g} / \mathrm{ml}$ did not exert cytotoxicity [8]. The cytotoxicity of SPIONs should therefore always be tested individually to determine the limits.
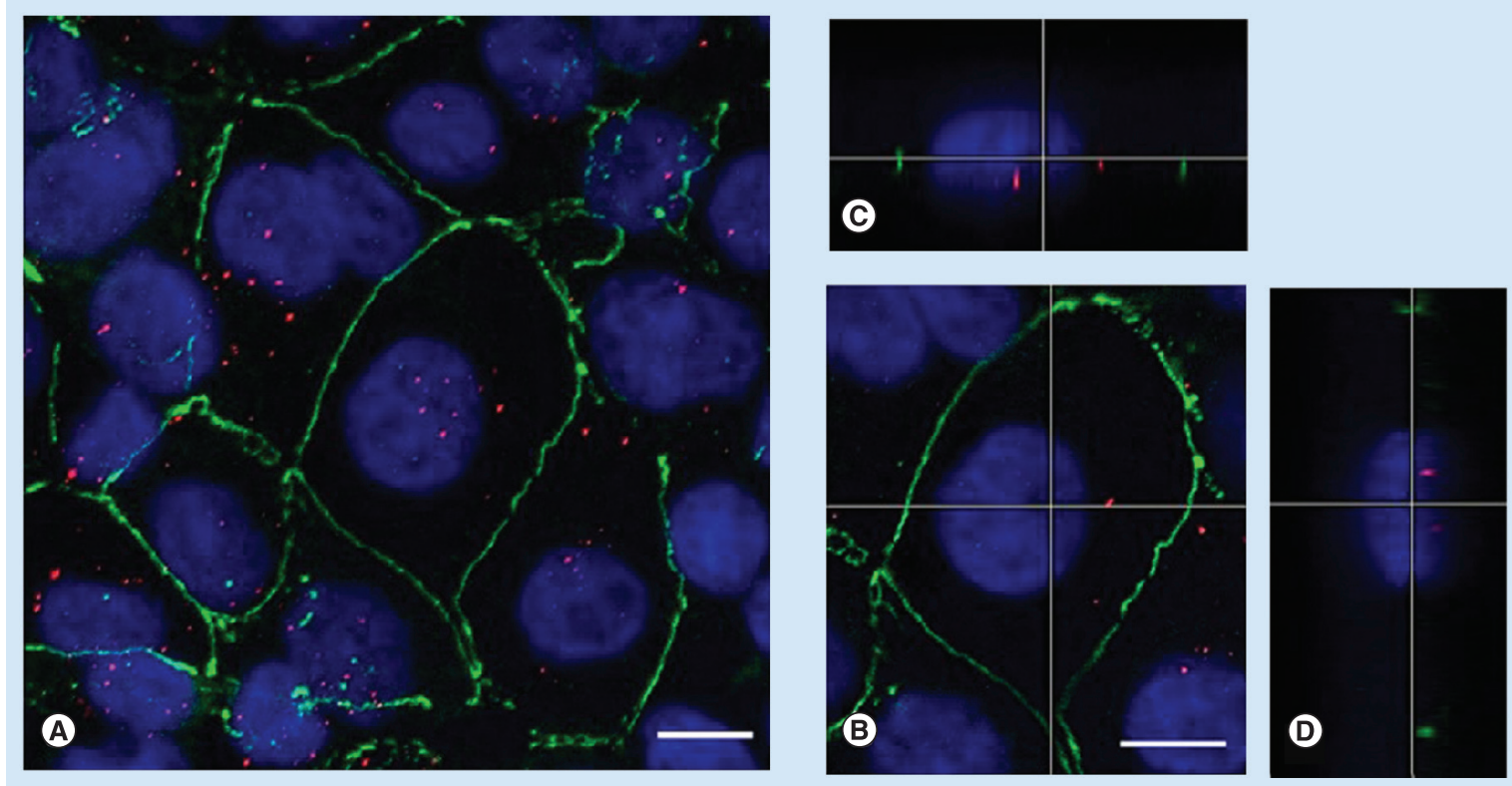

Figure 3. Uptake of magnetic nanoparticles at the blood-brain barrier in cultured brain capillary endothelial cells. Confocal images of BCECs stained for the tight junction protein ZO-1 (green) and nucleus (blue). SPIONs (140 $\mu$ g) were added to each insert with BCECs and incubated for $24 \mathrm{~h}$. The SPIONs (red) can be seen scattered around the inside of the cells: (A) a montage of a Z-stack of 25 slides, (B) a single slide in the Z-stack (X-Y plane) with focus on a single cell, (C) an X-Z plane of (B) and (D) a Y-Z plane of (B). Notably, SPIONs do not associate with the green color of ZO-1, indicating that they do not appear in the paracellular spaces between the BCECs. Likewise, the $Y-Z$ plane shows that the SPIONs are not distributed to the nucleus. The gray bars in (B) represent the site at which the $X-Z$ (C) and $Y-Z$ (D) sections are shown. The scale bar is $10 \mu \mathrm{m}[8]$.

Reproduced with permission from @ American Chemical Society (2013). 
Magnetic nanoparticles can be encapsulated in liposomes

The delivery of naked SPIONs to the brain has not received much attention so far. The entry of naked SPIONs is enhanced in brain tumors with a perturbed BBB [64]. Naked SPIONs are also able to passage brain endothelial cells in culture subjected to an external magnetic field [8]. Interestingly, many studies show the advantages of incorporating SPIONs into liposomes thus forming magneto-liposomes that are magnetic responsive [12,24,25,65-67]. Liposomes denote a group of well-established drug carriers for drug delivery purposes. They are formed in an aqueous solution using phospholipids or forming bilayers in a closed structure, with their hydrophilic head groups oriented outward toward the dispersant and inward toward an inner aqueous compartment [68]. The liposomes thereby have their hydrophobic tails of the phospholipids shielded from the outer and inner aqueous compartments. Liposomes are biocompatible and biodegradable and their size, composition and charge can be designed to fit the application profile. They can thus be loaded with hydrophobic cargos in their phospholipid bilayer and/or hydrophilic cargo in the inner aqueous compartment [68]. Liposomes are, however, just like SPIONs, prone to clearance by the RES. Therefore, PEG is often incorporated in the formulation of liposomes by using PEGylated phospholipids thereby displaying the PEG on the outer surface of the liposomes [68-70]. The liposomes can also be decorated with ligands or antibodies for targeting purposes, which often are conjugated to the distal end of PEGylated phosholipids to ensure that the ligand/antibody can interact with its target without steric hindrance from PEG [68-70].

The SPIONs can either be incorporated in the aqueous core of the liposomes, embedded in the liposomal membrane or both. Drugs can be carried by the magnetic liposomes either by being bound to the encapsulated SPIONs or by being co-encapsulated without binding to the SPIONs. The liposomes encapsulated SPIONs can thus be used as both a drug carrier that aids the liposomal drug delivery and as a contrast agent that aids guidance of the liposomes and ensures location specific drug delivery.

\section{Targeting the BBB using external magnetic force}

The application of an external magnetic force represents another and very different principle for targeted therapy to the brain when compared with the molecular targeted described above [8]. Magnetic nanoparticles are dragged though the BBB in vitro (Figure 4) [8], and theoretically, the force of the external magnet placed externally near the surface of the scull can be used to drag magnetic nanoparticles though the BBB, when these enter the cerebral microcirculation, as they may not otherwise pass the BBB [71-74]. The force of an external magnet drags magnetic nanoparticles in one direction toward the magnet as opposed to the magnetic field applied by MRI scanners. The force that a magnet can exert on SPIONs is dependent on the distance between the magnet and particle. The further away the lower the dragging force is. The working distance in humans is around $30-50 \mathrm{~cm}$ and a stronger magnet is therefore necessary compared with animals [9]. For human application the magnetic susceptibility of the SPIONs should therefore be very high to ensure responsiveness over long distances. In spite only few studies are reported, it can be postulated that the size of the magnetic nanoparticle dictates the extent to which they can affect the BBB, and that SPIONs and USPIONs are preferred as they exert low affection of the BBB integrity. These smaller magnetic nanoparticles are much easier to magnetize than the larger magnetic nanoparticles that are used as contrast agents for diagnostic purposes. It is speculated whether the 'pulling' forces of the magnetic field can harm the cells intended for uptake of the magnetic nanoparticles. Very large, $800 \mathrm{~nm}$ magnetic nanoparticles led to BBB leakage after systemic administration of silicone-coated magnetic nanoparticles to rats followed by application of a 0.4 Tesla magnetic field [62]. The large sized magnetic nanoparticles could have broken down the BBB when being dragged through the barrier thereby compromising its integrity. In culture, SPIONs with a size of $117 \mathrm{~nm}$ added to the endothelial cells combined with a magnetic field strength of 0.39 Tesla revealed that this magnetic force did not cause damage to the brain endothelial cells [8].

Polystyrene nanospheres with entrapped magnetic nanoparticles sized approximately $100 \mathrm{~nm}$ were able to cross the intact mouse BBB after intravenous injection by the aid of an externally applied magnet with a strength of 0.1 Tesla [9]. The application of the magnet increased the retention within the brain of the magnetic particles by approximately 25 -fold, and only minimal neurotoxicity was observed and no acute or long-term toxicity was detected [9]. When the external magnetic field was applied the magnetic polystyrene nanospheres not only entered brain endothelial cells, but they also passed into the perivascular space and brain parenchyma [9]. These observations were made on magnetic polystyrene nanospheres in brains vascularly perfused with TRICT-dextran, which only to some extent determined the distribution of the magnetic particles in the brain. Further studies using application of an external magnetic field should reveal the distribution of magnetic nanoparticles in the brain parenchyma, for 


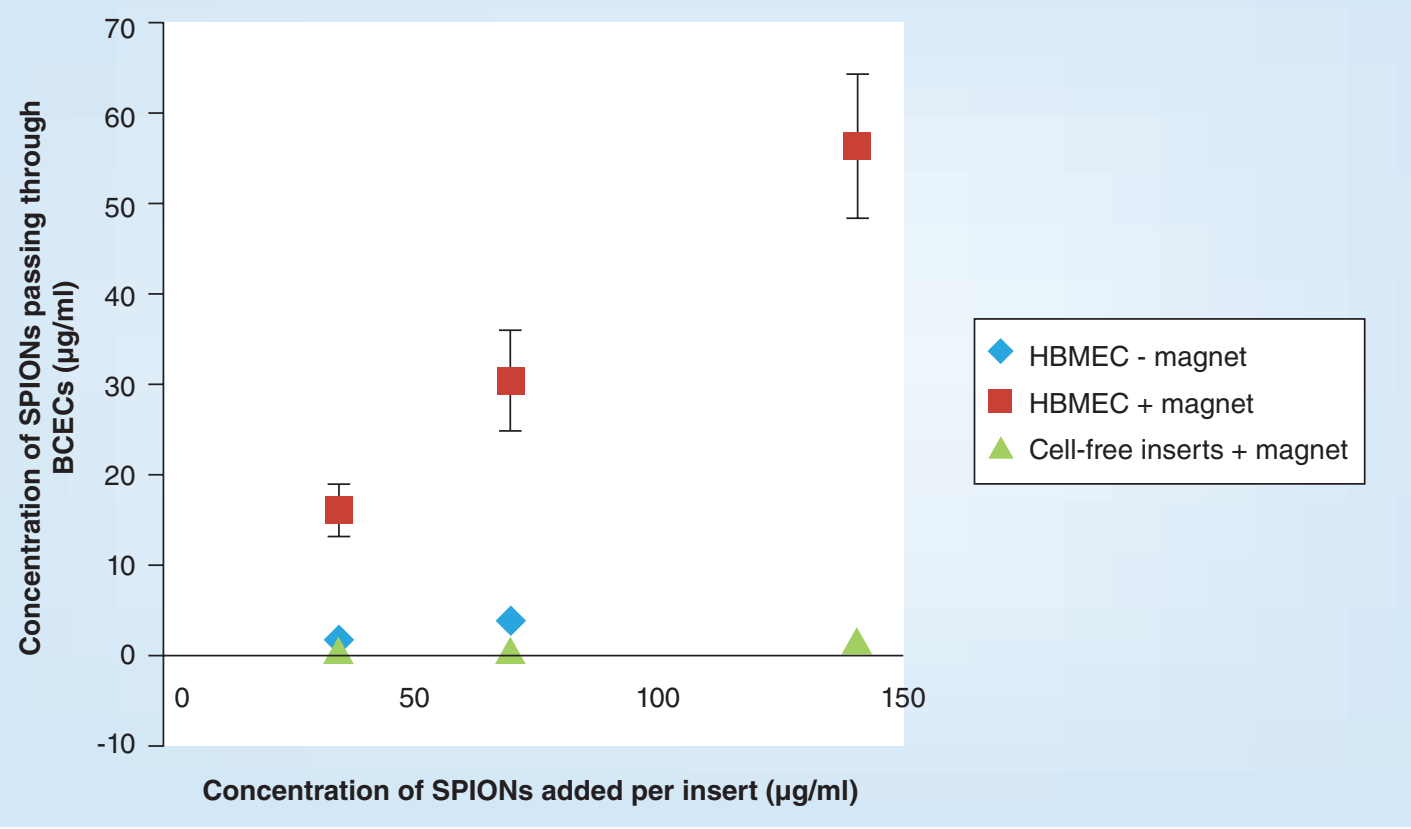

Figure 4. Quantitative assessment of the transport of magnetic nanoparticles through the blood-brain barrier in cultured brain capillary endothelial cells. Quantitative count between the concentrations of SPIONs added to inserts and their passage through BCECs in inserts and into wells containing astrocytes. Inserts with BCECs (red) and cell free inserts (green) that were both subjected to an external electric field for $5 \mathrm{~h}$ and inserts with BCECs (blue) devoid of exposure to an external electric force. SPIONs were added to BCECs at a concentration of 35, 70 or $140 \mu \mathrm{g} /$ insert. The amount of SPIONs passing the BCECs and entering into astrocytes was clearly larger when an external magnetic field was applied ( $p<0.001$ at either concentration added). There seems to be a linear correlation between the dose and response upon application of the magnetic field ( $n=4$ observations per point at 70 and $140 \mu \mathrm{g}$, and $\mathrm{n}=3$ observations per point at $35 \mu \mathrm{g}$ [results are means $\pm \mathrm{SE}$ ]) [8]. Reproduced with permission from (C) American Chemical Society (2013).

example, by colocalization with hallmark markers of neurons and astrocytes [9].

The utility of SPIONs may also improve considering the potential of encapsulating magnetic nanoparticles inside drug carriers such as liposomes, which simultaneously can carry considerable amounts of therapeutic molecules. Magneto-liposomes show great potential for $\mathrm{BBB}$ passage leading to subsequent drug delivery to the brain. Transferrin-coated PEGylated magneto-liposomes sized approximately $130 \mathrm{~nm}$ showed improved passage through a human BBB in vitro by up to $100 \%$ when applying a magnetic force of 0.08 Tesla [24]. The barrier integrity was intact after magnetic force application and no significant cytotoxicity was observed [24]. Magneto-liposomes have also been used for in vitro delivery of an antiretroviral therapeutics across the BBB [25]. 3'Azido-3'deoxythymidine-5'-triphosphate (AZTTP) were bound to magnetic particles $(35 \mathrm{~nm})$ and encapsulated in liposomes [25]. The AZTTPmagneto-liposomes had an average size of $150 \mathrm{~nm}$ and were able to cross an in vitro human BBB model by the aid of an external magnetic field of 0.3 Tesla without harming the BBB [25]. The permeability of AZTTP magneto-liposomes was threefold higher than free AZTTP. Magneto-liposomes have also been used for increasing monocyte drug delivery across the BBB by more than twofold with the aid of an applied magnetic field strength of 0.3 Tesla [25].

\section{Stepwise targeting of magnetic}

nanoparticles \& application of an external magnetic field could enable enhanced drug transport to the brain

Based on the current assumptions on how targeted delivery can occur at the $\mathrm{BBB}$, it is justifiable to propose a two-step mechanism suggesting how magnetic nanoparticles co-encapsulated with therapeutic molecules can undergo further transport into the brain (Figure 5):

- Step 1: Blood-to-brain endothelium transport: drug carriers co-encapsulated with magnetic nanoparticles and therapeutic molecules are targeted to the BBB using conjugation on their outer surface with antibodies with molecules expressed by brain endothelial cells like the transferrin receptor. 


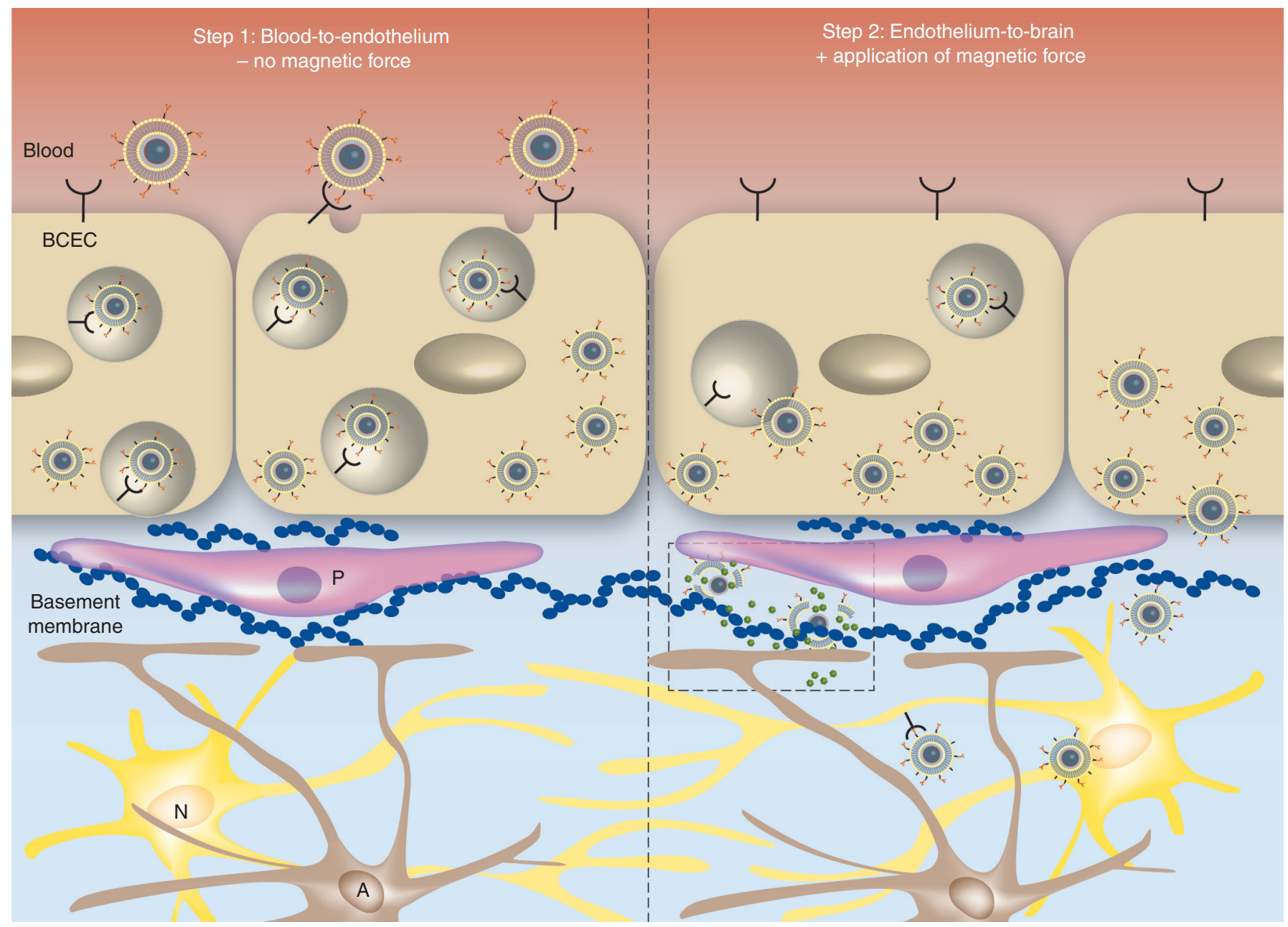

Figure 5. Illustration showing a possible step-wise transport of targeted drug carriers containing both magnetic nanoparticles and therapeutic molecules though the blood-brain barrier and beyond. Step 1: Blood-to-brain endothelium transport: drug carriers co-encapsulated with magnetic nanoparticles and therapeutic molecules are targeted to the transferrin receptor on brain capillary endothelial cells. Step 2: Endothelium-to-brain transport: Once accumulated inside the brain endothelium, magnetic force is applied externally on the cranial surface, which leads to subsequent dragging of the drug carrier through the brain endothelial cells, pass the NVU including its basal membrane. This leads to the occurrence of the drug carrier inside the brain from where the therapeutic molecules can be released from the drug carrier and enable neuronal targeting. The perforated square illustrates the release of therapeutic molecules (green) from degraded magneto-liposomes in the brain.

A: Astrocyte; BM: Basement membrane; P: Pericyte.

- Step 2: Endothelium-to-brain transport: once accumulated inside the brain endothelium, magnetic force is applied externally on the cranial surface. This leads to subsequent dragging of the magnetic nanoparticle through the brain endothelial cells and pass the NVU including its basal membrane. This leads to the occurrence of the drug carrier inside the brain from where the therapeutic molecules can be released from the drug carrier and enable neuronal targeting. In vitro studies of the toxicology of magnetic particles show that the particles are harmless to brain endothelial cells and that the transport through their cellular membranes occurs without significant impairment of their integrity. A so far unraveled question concerns the aspect of whether only the magnetic nanoparticle undergoes transport through the brain endothelial cells under the influence of the magnetic nanoparticle, while leaving the remaining part of the drug carrier inside the brain endothelial cell. Further studies are necessary to address this matter, which also applies to the possible fate of the drug carrier being entrapped inside the basal membrane at the NVU. Issues related to accumulation of magnetic nanoparticles at the BBB or the adjacent NVU may probably be overcome via optimizing issues, for example, distance and field strength related to the application of the external magnetic force. 


\section{Future perspective}

In this review we have described how we believe that magnetic nanoparticles would add new dimensions to drug delivery to the brain. Addition of magnetic nanoparticles in decorated and functionalized, cargocontaining liposomes would enable double targeting, in other words, cell-specific targeting aided by, for example, antitransferrin antibody and area specific targeting aided by an external magnetic field.

The issues with toxicity occurring when magnetic nanoparticles are degraded could be limited by the focused application of a magnetic field, and thereby decreasing the amount of applied magnetic nanoparticles. Issues of how magnetic nanoparticles can be applied in the clinic needs to be addressed in the future. Issues such as how blood flow and blood vessel orientation will influence the strength of the applied magnetic field need further investigations. Furthermore, the size of the brain and its coverings in animals are quite different from humans and should therefore also be addressed in studies on how these issues impact the magnetic force and the delivery of magnetically guided drug carriers.

\section{Financial \& competing interests disclosure}

The authors have no relevant affiliations or financial involvement with any organization or entity with a financial interest in or financial conflict with the subject matter or materials discussed in the manuscript. This includes employment, consultancies, honoraria, stock ownership or options, expert testimony, grants or patents received or pending, or royalties.

No writing assistance was utilized in the production of this manuscript.

\section{Open access}

This work is licensed under the Attribution-NonCommercialNoDerivatives 4.0 Unported License. To view a copy of this license, visit http://creativecommons.org/licenses/by-nc-nd/4.0

\section{Executive summary}

- Drug delivery to the brain can be enhanced by the transport of magnetic nanoparticles across the blood-brain barrier aided by an extracranially applied magnetic force.

- Magnetic nanoparticles can be encapsulated in liposomes and carry large molecules with therapeutic, for example, siRNA, cDNA and polypeptides.

- Conjugation of nanoparticles with antibodies that target molecules expressed by brain capillary endothelial cells may facilitate their uptake and transport into the brain.

- Stepwise targeting of liposome encapsulated magnetic nanoparticles to brain capillary endothelial cells followed by magnetically guided transport through the blood-brain barrier using an external magnetic force may prove a novel mechanism for targeted therapy of macromolecules to the brain.

\section{References}

Papers of special note have been highlighted as: • of interest;

• of considerable interest

1 Abbott NJ, Patabendige AAK, Dolman DEM, Yusof SR, Begley DJ. Structure and function of the blood-brain barrier. Neurobiol. Dis. 37(1), 13-25 (2010).

2 Cardoso FL, Brites D, Brito MA. Looking at the bloodbrain barrier: molecular anatomy and possible investigation approaches. Brain Res. Rev. 64(2), 328-363 (2010).

3 Masserini M. Nanoparticles for brain drug delivery. ISRN Biochem. 2013, 238428 (2013).

4 Gabathuler R. Approaches to transport therapeutic drugs across the blood-brain barrier to treat brain diseases. Neurobiol. Dis. 37(1), 48-48-57 (2010).

5 Lichota J, Skjørringe T, Thomsen LB, Moos T. Macromolecular drug transport into the brain using targeted therapy. J. Neurochem. 113(1), 1-13 (2010).

6 Walsh G. Biopharmaceuticals: Biochemistry and Biotechnology (Second Edition). John Wiley \& Sons Ltd., ISBN 978-0-47084326-0 (2003).

7 Watts RJ, Dennis MS. Bispecific antibodies for delivery into the brain. Curr. Opin. Chem. Biol. 17(3), 393-399 (2013).
8 Thomsen LB, Linemann T, Pondman KM et al. Uptake and transport of superparamagnetic iron oxide nanoparticles through human brain capillary endothelial cells. ACS Chem. Neurosci. 4(10), 1352-1360 (2013).

- In vitro study on brain delivery of magnetic nanoparticles by the aid of an externally applied magnetic force.

9 Kong SD, Lee J, Ramachandran S et al. Magnetic targeting of nanoparticles across the intact blood-brain barrier. J. Control Release 164(1), 49-57 (2012).

-• In vivo study on brain delivery of magnetic nanoparticles by the aid of an externally applied magnetic force.

10 Sagar V, Pilakka-Kanthikeel S, Atluri VS et al. Therapeutical neurotargeting via magnetic nanocarrier: implications to opiate-induced neuropathogenesis and NeuroAIDS. J. Biomed. Nanotechnol. 11, 1-12 (2015).

11 Sagar V, Pilakka-Kanthikeel S, Pottathil R, Saxena SK, Nair M. Towards nanomedicines for neuroAIDS. Rev. Med. Virol. 24(2), 103-124 (2014).

12 Carvalho A, Martins MBF, Corvo ML, Feio G. Enhanced contrast efficiency in MRI by PEGylated magnetoliposomes loaded with PEGylated SPION: effect of SPION coating and micro-environment. Mater. Sci. Eng. C Biol. Appl. 43, 521-521-6 (2014).

13 Verma J, Lal S, Van Noorden CJF. Nanoparticles for 
hyperthermic therapy: synthesis strategies and applications in glioblastoma. Int. J. Nanomedicine 9, 2863-2863-77 (2014).

14 Ruan J, Shen J, Wang Z et al. Efficient preparation and labeling of human induced pluripotent stem cells by nanotechnology. Int. J. Nanomedicine 6, 425-435 (2011).

15 Gordon R, Hogan CE, Neal ML, Anantharam V, Kanthasamy AG, Kanthasamy A. A simple magnetic separation method for high-yield isolation of pure primary microglia. J. Neurosci. Methods 194(2), 287-296 (2011).

16 Madsen SJ, Gach HM, Hong SJ, Uzal FA, Peng Q, Hirschberg H. Increased nanoparticle-loaded exogenous macrophage migration into the brain following PDT-induced blood-brain barrier disruption. Lasers Surg. Med. 45(8), 524-532 (2013).

17 Scherer F, Anton M, Schillinger U et al. Magnetofection: enhancing and targeting gene delivery by magnetic force in vitro and in vivo. Gene Ther. 9(2), 102-109 (2002).

18 Zhao M, Li A, Chang J et al. Develop a novel superparamagnetic nano-carrier for drug delivery to brain glioma. Drug Deliv. 20(3-4), 95-101 (2013).

19 Boyer C, Whittaker MR, Bulmus V, Liu J, Davis TP. The design and utility of polymer-stabilized iron-oxide nanoparticles for nanomedicine applications. NPG Asia Mater. 2, 23-30 (2010).

20 Pilakka-Kanthikeel S, Atluri VSR, Sagar V, Saxena SK, Nair M. Targeted brain derived neurotropic factors (BDNF) delivery across the blood-brain barrier for neuro-protection using magnetic nano carriers: an in-vitro study. PLoS ONE 8(4), e62241-e62241 (2013).

21 Shevtsov MA, Nikolaev BP, Yakovleva LY et al. Superparamagnetic iron oxide nanoparticles conjugated with epidermal growth factor (SPION-EGF) for targeting brain tumors. Int. J. Nanomedicine 9, 273-87 (2014).

22 Halamoda Kenzaoui B, Angeloni S, Overstolz T et al. Transfer of ultrasmall iron oxide nanoparticles from human brain-derived endothelial cells to human glioblastoma cells. ACS Appl. Mater. Interfaces 5(9), 3581-3586 (2013).

23 Varallyay CG, Nesbit E, Fu R et al. High-resolution steady-state cerebral blood volume maps in patients with central nervous system neoplasms using ferumoxytol, a superparamagnetic iron oxide nanoparticle. J. Cereb. Blood. Flow. Metab. 33(5), 780-786 (2013).

24 Ding H, Sagar V, Agudelo M et al. Enhanced bloodbrain barrier transmigration using a novel transferrin embedded fluorescent magneto-liposome nanoformulation. Nanotechnology 25(5), 055101-055101 (2014).

- $\quad$ BBB passage of liposomes is enhanced by addition of both transferrin receptor targeting and magnetic nanoparticles.

25 Saiyed Z, Gandhi N, Nair MPN. Magnetic nanoformulation of azidothymidine 5'-triphosphate for targeted delivery across the blood-brain barrier. Int. J. Nanomedicine 5, 157-166 (2010).

26 Burkhart A, Azizi M, Thomsen MS, Thomsen LB, Moos T. Accessing targeted nanoparticles to the brain: the vascular route. Curr. Med. Chem. 21(36), 4092-4099 (2014).

27 Abbott NJ, Rönnbäck L, Hansson E. Astrocyte-endothelial interactions at the blood-brain barrier. Nat. Rev. Neurosci. 7(1), 41-53 (2006).

28 Armulik A, Genove G, Mäe M et al. Pericytes regulate the blood-brain barrier. Nature 468(7323), 557-561 (2010).

29 Daneman R, Zhou L, Kebede AA, Barres BA. Pericytes are required for blood-brain barrier integrity during embryogenesis. Nature 468(7323), 562-566. (2010).

30 Armulik A, Genové G, Betsholtz C. Pericytes: developmental, physiological, and pathological perspectives, problems, and promises. Dev. Cell 21(2), 193-193-215 (2011).

31 Gosk S, Vermehren C, Storm G, Moos T. Targeting antitransferrin receptor antibody (OX26) and OX26-conjugated liposomes to brain capillary endothelial cells using in situ perfusion. J. Cereb. Blood Flow Metab. 24(11), 1193-1204 (2004).

32 Muldoon LL, Pagel MA, Kroll RA, Roman-Goldstein S, Jones RS, Neuwelt EA. A physiological barrier distal to the anatomic blood-brain barrier in a model of transvascular delivery. AJNR Am. J. Neuroradiol. 20 (2), 217-222 (1999).

33 Engelhardt B, Sorokin L. The blood-brain and the bloodcerebrospinal fluid barriers: function and dysfunction. Semin. Immunopathol. 31(4), 497-511 (2009).

34 Timpl R. Structure and biological activity of basement membrane proteins. Eur. J Biochem. 180 (3), 487-502 (1989).

35 Mathiisen TM, Lehre KP, Danbolt NC, Ottersen OP. The perivascular astroglial sheath provides a complete covering of the brain microvessels: an electron microscopic 3D reconstruction. Glia 58(9), 1094-1103 (2010).

36 Yu YJ, Atwal JK, Zhang Y et al. Therapeutic bispecific antibodies cross the blood-brain barrier in nonhuman primates. Sci. Transl. Med. 6(261) 261ra154 (2014).

37 Yu YJ, Zhang Y, Kenrick M et al. Boosting brain uptake of a therapeutic antibody by reducing its affinity for a transcytosis target. Sci. Transl. Med. 3(84) 84 ra44 (2011).

38 Niewoehner J, Bohrmann B, Collin L et al. Increased brain penetration and potency of a therapeutic antibody using a monovalent molecular shuttle. Neuron 81(1), 49-60 (2014).

39 Rapoport SI. Osmotic opening of the blood-brain barrier. Ciba Found. Symp. 56, 237-55 (1978).

40 Dallas S, Miller DS, Bendayan R. Multidrug resistanceassociated proteins: expression and function in the central nervous system. Pharmacol. Rev. 58(2), 140-140-61 (2006).

41 Jefferies WA, Brandon MR, Hunt SV, Williams AF, Gatter KC, Mason DY. Transferrin receptor on endothelium of brain capillaries. Nature 312 (5990), 162-163 (1984).

42 Friden PM, Walus LR, Musso GF, Taylor MA, Malfroy B, Starzyk RM. Anti-transferrin receptor antibody and antibody-drug conjugates cross the blood-brain barrier. Proc. Natl Acad. Sci. USA 88(11), 4771-4775 (1991).

43 Moos T, Morgan EH. Restricted transport of anti-transferrin receptor antibody (OX26) through the blood-brain barrier in the rat. J. Neurochem. 79(1), 119-129 (2001).

44 Paris-Robidas S, Emond V, Tremblay C, Soulet D, Calon F. In vivo labeling of brain capillary endothelial cells after intravenous injection of monoclonal antibodies targeting the transferrin receptor. Mol. Pharmacol. 80(1), 32-39 (2011). 
45 Moos T, Rosengren Nielsen T, Skjørringe T, Morgan EH. Iron trafficking inside the brain. J. Neurochem. 103(5), 1730-1740 (2007).

Moos T, Oates PS, Morgan EH. Expression of the neuronal transferrin receptor is age dependent and susceptible to iron deficiency. J. Comp. Neurol. 398(3), 420-430 (1998).

47 Skjørringe T, Burkhart A, Johnsen KB, Moos T. Divalent metal transporter 1 (DMT1) in the brain: implications for a role in iron transport at the blood-brain barrier, and neuronal and glial pathology. Front. Mol. Neurosci. 8, 19 (2015).

48 Abbott NJ. Blood-brain barrier structure and function and the challenges for CNS drug delivery. J. Inherit. Metab. Dis. 36(3), 437-437-49 (2013).

49 Wadghiri YZ, Li J, Wang J et al. Detection of amyloid plaques targeted by bifunctional USPIO in Alzheimer's disease transgenic mice using magnetic resonance microimaging. PLoS ONE 8(2), e57097 (2013).

50 Fan C, Ting C, Lin $\mathrm{H}$ et al. SPIO-conjugated, doxorubicinloaded microbubbles for concurrent MRI and focusedultrasound enhanced brain-tumor drug delivery. Biomaterials 34(14), 3706-3715 (2013).

51 Wahajuddin, Arora S. Superparamagnetic iron oxide nanoparticles: magnetic nanoplatforms as drug carriers. Int. J. Nanomedicine 7, 3445-3471 (2012).

52 Mok H, Zhang M. Superparamagnetic iron oxide nanoparticle-based delivery systems for biotherapeutics. Expert Opin. Drug Deliv. 10(1), 73-87 (2013).

53 Denizot B, Tanguy G, Hindre F, Rump E, Le Jeune J, Jallet P. Phosphorylcholine coating of iron oxide nanoparticles. J. Colloid Interface Sci. 209(1), 66-71 (1999).

54 Kumar A, Jena P, Behera S, Lockey R, Mohapatra S. Multifunctional magnetic nanoparticles for targeted delivery. Nanomedicine 6(1), 64-69 (2010).

55 Chertok B, Moffat B, David A et al. Iron oxide nanoparticles as a drug delivery vehicle for MRI monitored magnetic targeting of brain tumors. Biomaterials 29(4), 487-496 (2008).

56 Moghimi SM, Hunter AC, Murray JC. Long-circulating and target-specific nanoparticles: theory to practice. Pharmacol. Rev. 53(2), 283-318 (2001).

57 Yallapu M, Foy S, Jain T, Labhasetwar V. PEGfunctionalized magnetic nanoparticles for drug delivery and magnetic resonance imaging applications. Pharm. Res. 27(11), 2283-2295 (2010).

58 Dias AMGC, Hussain A, Marcos AS, Roque ACA. A biotechnological perspective on the application of iron oxide magnetic colloids modified with polysaccharides. Biotechnol. Adv. 29(1), 142-155 (2011).

59 Naqvi S, Samim M, Abdin M et al. Concentrationdependent toxicity of iron oxide nanoparticles mediated by increased oxidative stress. Int. J. Nanomedicine 5, 983-989 (2010).

60 Sun Z, Yathindranath V, Worden M et al. Characterization of cellular uptake and toxicity of aminosilane-coated iron oxide nanoparticles with different charges in central nervous system-relevant cell culture models. Int. J. Nanomedicine 8 , 961-970 (2013).
61 Schaub NJ, Rende D, Yuan Y, Gilbert RJ, Borca-Tasciuc D. Reduced astrocyte viability at physiological temperatures from magnetically activated iron oxide nanoparticles. Chem. Res. Toxicol. 27(12), 2023-35 (2014).

62 Stepp P, Thomas F, Lockman PR, Chen H, Rosengart AJ. In vivo interactions of magnetic nanoparticles with the blood-brain barrier. J. Magn. Magn. Mater. 321(10), 1591-1593 (2009).

63 Mahmoudi M, Laurent S, Shokrgozar MA, Hosseinkhani $M$. Toxicity evaluations of superparamagnetic iron oxide nanoparticles: cell 'vision' versus physicochemical properties of nanoparticles. ACS Nano. 5, 7263-7276 (2011).

- Addresses how cytotoxicity is influenced by surface coat, surface charge and cell-specific response.

64 Chen Y, Chiang C, Wu S, Hsieh W, Lin W. Targeting microbubbles-carrying TGF $\beta 1$ inhibitor combined with ultrasound sonication induce $\mathrm{BBB} / \mathrm{BTB}$ disruption to enhance nanomedicine treatment for brain tumors. J. Control. Release 211, 53-62 (2015).

65 Zhao M, Chang J, Fu X et al. Nano-sized cationic polymeric magnetic liposomes significantly improves drug delivery to the brain in rats. J. Drug Target. 20 (5), 416-41621 (2012)

66 Amstad E, Kohlbrecher J, Müller E, Schweizer T, Textor $\mathrm{M}$, Reimhult E. Triggered release from liposomes through magnetic actuation of iron oxide nanoparticle containing membranes. Nano Lett. 11(4), 1664-1670 (2011).

67 Martina M, Fortin J, Ménager C et al. Generation of superparamagnetic liposomes revealed as highly efficient MRI contrast agents for in vivo imaging. J. Am. Chem. Soc. 127(30), 10676-10685 (2005).

68 Paszko E, Senge MO. Immunoliposomes. Curr. Med. Chem. 19(31), 5239-5277 (2012).

69 Jølck RI, Feldborg LN, Andersen S, Moghimi SM, Andresen TL. Engineering liposomes and nanoparticles for biological targeting. Adv. Biochem. Eng. Biotechnol. 125, 251-280 (2011).

70 Samad A, Sultana Y, Aqil M. Liposomal drug delivery systems: an update review. Curr. Drug Deliv. 4(4), 297-305 (2007).

71 Hoff D, Sheikh L, Bhattacharya S, Nayar S, Webster TJ. Comparison study of ferrofluid and powder iron oxide nanoparticle permeability across the blood-brain barrier. Int J. Nanomedicine 8, 703-710 (2013).

72 Cintra e Silva Débora de Oliveira, Estevanato LLC, Simioni AR et al. Successful strategy for targeting the central nervous system using magnetic albumin nanospheres. J. Biomed. Nanotechnol. 8(1), 182-189 (2012).

73 Qiao R, Jia Q, Hüwel S et al. Receptor-mediated delivery of magnetic nanoparticles across the blood-brain barrier. ACS Nano 6(4), 3304-3310 (2012).

74 Dilnawaz F, Singh A, Mewar S, Sharma U, Jagannathan NR, Sahoo SK. The transport of non-surfactant based paclitaxel loaded magnetic nanoparticles across the blood brain barrier in a rat model. Biomaterials 33(10), 2936-2951 (2012). 